\title{
Les facteurs de l'organisation spatiale des écoulements en étiage. Exemple du bassin versant du Mouzon (Lorraine)
}

Spatial organisation factors of low water periods. Example of the Mouzon basin (Lorraine)

Die Faktoren der räumlichen Organisation der Abflüsse bei Niedrigwasser.

Beispiel des Beckens des Mouzon (Lothringen)

Jeanine Corbonnois

\section{(2) OpenEdition}

\section{Journals}

Édition électronique

URL : http://journals.openedition.org/rge/4165

DOI : $10.4000 /$ rge.4165

ISSN : $2108-6478$

Éditeur

Association des géographes de l'Est

Édition imprimée

Date de publication : 1 janvier 2000

ISSN : 0035-3213

\section{Référence électronique}

Jeanine Corbonnois, «Les facteurs de l'organisation spatiale des écoulements en étiage. Exemple du bassin versant du Mouzon (Lorraine) », Revue Géographique de l'Est [En ligne], vol. 40 / 1-2 | 2000, mis en ligne le 05 août 2013, consulté le 08 septembre 2020. URL : http://journals.openedition.org/rge/ 4165 ; DOI : https://doi.org/10.4000/rge.4165

Ce document a été généré automatiquement le 8 septembre 2020

Tous droits réservés 


\section{Les facteurs de l'organisation spatiale des écoulements en étiage. Exemple du bassin versant $d u$ Mouzon (Lorraine)}

Spatial organisation factors of low water periods. Example of the Mouzon basin

(Lorraine)

Die Faktoren der räumlichen Organisation der Abflüsse bei Niedrigwasser.

Beispiel des Beckens des Mouzon (Lothringen)

Jeanine Corbonnois

1 En étiage, le débit des cours d'eau est déterminé par les conditions du climat, la configuration du substratum géologique, la géomorphologie et les caractéristiques du couvert végétal. Dans un bassin versant, ce débit parfois très variable (Zumstein 1976, Maire 1977), peut être analysé à partir de mesures, effectuées sur le réseau hydrographique pour caractériser le rendement en eau du substratum, lorsque les précipitations n'influencent plus directement les écoulements. Ce rendement est commandé par le volume et la dynamique des aquifères, révélés par les variations spatiales du débit des cours d'eau (Sary, 1993), mais également par la teneur de l'eau en éléments dissous, signature du réservoir qui alimente les écoulements de surface.

2 Le bassin du Mouzon, qui montre une grande hétérogénéité lithologique, nous permet d'observer comment s'organisent les écoulements à différents moments de l'étiage selon les apports hydrogéologiques et leur dynamique. En comparant les caractéristiques des quatre cours d'eau principaux qui drainent ce bassin : Bani, Anger, ruisseau de l'Étang, et Mouzon, il est possible de déterminer et de hiérarchiser les facteurs qui influencent spatialement cette organisation. 


\section{Objectif et méthode}

3 L'étude des étiages se rapporte souvent à l'analyse de la baisse du débit pendant la période de basses eaux, à l'exutoire de bassins versants (Dacharry, 1975). Le fonctionnement hydrologique est alors appréhendé au cours du temps, en particulier en phase de tarissement (Rambert, 1971, Lambert, 1994), influencée par l'hydrogéologie (Castany, 1982). L'analyse révèle l'existence d'un ou de plusieurs aquifères, livrant alors de l'eau au réseau hydrographique par l'intermédiaire de sources (Merot, 1988). Ces approches sont menées dans le but de définir les écoulements de base par rapport aux écoulements de crue, pour réaliser des bilans hydrologiques (Cosandey, 1983) ou déterminer le volume des réserves en eau souterraines. Dans le bassin du Mouzon, c'est la détermination de l'organisation spatiale des écoulements à l'intérieur du bassin versant qui a été privilégiée.

Des travaux relatifs aux débits d'étiage et de basses eaux sont menés depuis de nombreuses années par le Centre d'Etudes Géographiques de l'Université de Metz. Ils ont abouti à l'élaboration d'une méthode en vue de revaloriser le catalogue des débits d'étiage de l'Agence de l'Eau Rhin-Meuse (François et al., 1994).

Cette méthode repose sur la construction de profils hydrologiques obtenus en corrélant la surface de bassin-versant mesurée à différents points d'un cours d'eau avec le débit correspondant. Les mesures, répétées lors de plusieurs campagnes de jaugeage, permettent par les profils hydrologiques, de déterminer les conditions de l'alimentation du réseau hydrographique et leur évolution dans l'espace, pour les situations hydrologiques différentes. Par cette méthode, il est possible de localiser les principaux apports d'eau livrés par les bassins versants, d'identifier les réservoirs qui y participent et de déterminer leur capacité à soutenir les débits en étiage.

6 Lorsque ces apports diminuent dans tout le bassin, à la même vitesse pendant l'étiage, les profils obtenus, composés en coordonnées logarithmiques d'un ou de plusieurs segments de droite, restent parallèles. C'est le cas pour des cours d'eau qui drainent les Vosges et leurs abords, où existent des réservoirs souterrains (grès vosgiens) puissants et abondamment alimentés par les précipitations (Arts et François, 1998). Mais pour des cours d'eau qui drainent des régions de plateau moins arrosées, où les réservoirs souterrains ont des capacités de stockage d'eau variables, le parallélisme des profils est altéré par le tarissement précoce ou tardif des apports du substratum, selon le fonctionnement hydrodynamique des aquifères.

7 Ce mode de fonctionnement est celui du bassin du Mouzon. La variation des débits est représentée sur les profils hydrologiques et sur des cartes de spatialisation des écoulements. Celles-ci font apparaître le rétrécissement des aires d'alimentation du réseau hydrographique lorsque la sévérité de l'étiage augmente, et que seuls les réservoirs majeurs restent productifs. Pour confirmer l'origine de l'eau, nous avons utilisé un traceur hydochimique simple à déterminer (Martin, 1991). La dureté de l'eau, exprimée en équivalent $\mathrm{CaCO} 3 \mathrm{mg} / \mathrm{l}$, suffit en effet pour identifier les principaux aquifères selon les grands ensembles lithologiques qui affleurent dans le bassin versant (Lelong et al., 1994).

8 Cette méthode qui repose sur l'interprétation des données collectées à l'intérieur du bassin versant, est assez lourde à mettre en œuvre. Mais elle permet une bonne 
appréciation des potentialités hydrogéologiques du bassin et des facteurs explicatifs de l'étiage.

\section{Les données utilisées}

\section{A. Quelques caractéristiques du bassin du Mouzon}

Le bassin de Mouzon, très composite, fournit un bon exemple pour analyser la distribution des apports d'eau dans l'espace au cours de l'étiage. D'une superficie de 415 $\mathrm{km} 2$, il est inscrit dans les plateaux du Sud-Ouest de la Lorraine et drainé par un réseau hydrographique dont les artères principales (Mouzon, Anger et ruisseau de l'Étang, Bani), confluent dans la partie aval du bassin, avant de rejoindre en rive droite, la Meuse à Neufchâteau. Ce réseau hydrographique généralement conséquent, traverse, selon une direction SSE/NNW, les assises sédimentaires (depuis le Muschelkalk, Trias moyen, jusqu'au Bajocien inférieur), composées alternativement de calcaire, de marnes et de grès. Les couches, doucement inclinées vers l'Ouest, arment plusieurs cuestas de direction SW/NE, que les cours d'eau traversent perpendiculairement (Fig. 1). Le Mouzon draine la moitié occidentale du bassin. L'autre moitié est parcourue par l'Anger, le ruisseau de l'Etang et le Bani.

Fig. 1 : Lithostratigraphie et formes principales dans le bassin du Mouzon
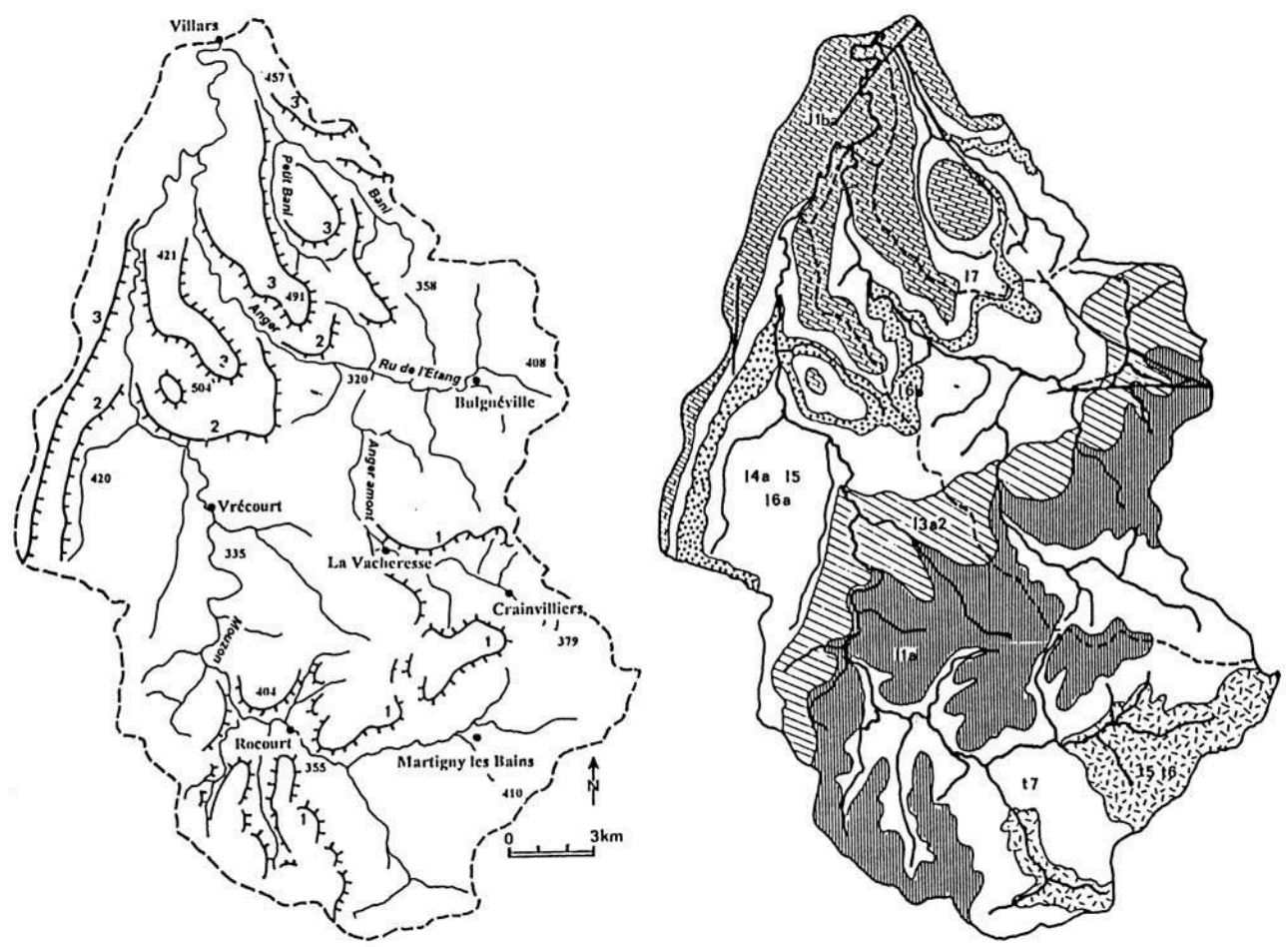

1 : Côte de l'Infra-Lias ; 2 : Côte des grès médioliasiques ; 3 : Côte du Bajocien

$\mathrm{J} 1 \mathrm{ba}$ : Calcaires du Bajocien ; 17 : Marnes du Toarcien ; I 6b : Grès médioliasiques ; | 4a, I 5, I 6a : Marnes du Domérien et marno-calcaires du Lias Moyen ; I 3a2 : Calcaires à gryphées ; I 1a : Grès du Rhétien ; t 7 : Argiles du Keuper ; 5 et $t 6$ : Calcaires du Muschelkalk

Le bassin du Mouzon est situé dans une région rurale. Un tiers des surfaces est occupé par des forêts, situées sur le revers des cuestas. Le reste est partagé entre les secteurs 
toujours en herbe qui occupent toute la partie centrale du bassin, et des labours. Il comprend quelques villages, dont les plus importants sont localisés à l'amont du bassin (Bulgnéville, Martigny-les-Bains).

Le débit du Mouzon est mesuré à la station de Villars située juste à l'aval de la confluence du Bani, qui contrôle une surface de $405 \mathrm{~km} 2$. Le module est de 4,82 m3/s $(11,9 \mathrm{l} / \mathrm{s} / \mathrm{km} 2)$, soit $375 \mathrm{~mm}$ de lame d'eau écoulée par an (période $74 / 95)$, pour des précipitations annuelles de l'ordre de $1000 \mathrm{~mm}$ (François et al., 1993). Son régime est de type pluvial océanique (maximum hivernal: $10 \mathrm{~m} 3 / \mathrm{s}$ en décembre et janvier, minimum estival : $0,3 \mathrm{~m} 3 / \mathrm{s}$ en août). Comme pour la plupart des rivières lorraines, des variations importantes peuvent être observées en fonction des précipitations. Le débit moyen annuel varie entre $1,87 \mathrm{~m} 3 / \mathrm{s}$ (tranche écoulée de $145 \mathrm{~mm}$ en 1976) et $6,5 \mathrm{~m} 3 / \mathrm{s}$ (506 mm en 1983); à l'échelle mensuelle, il peut dépasser $20 \mathrm{~m} 3 / \mathrm{s}$ (janvier 1995, décembre 1981) et s'abaisser à moins de $100 \mathrm{l} / \mathrm{s}$ (août 1976, août 1983). Dans le bassin du Mouzon les écarts sont exagérés par le faible effet tampon du substratum: roches imperméables étendues, roches perméables peu capacitives en raison de leur minceur (15 à $30 \mathrm{~m}$ de grès rhétiens, grès médioliasiques, calcaires à gryphées) ou de leur grande fissuration (calcaires du Bajocien). Les variations mesurées au cours de l'année 1983, assez arrosée (précipitations annuelles : $1077 \mathrm{~mm}$ ), fournissent une bonne illustration $\mathrm{du}$ comportement du bassin dont le caractère ruisselant impose aux écoulements un long cheminement en surface et une exposition aux prélèvements évaporatoires. Au cours de cette année, le débit s'est abaissé de $23,7 \mathrm{~m} 3 / \mathrm{s}$ en mai à $0,032 \mathrm{~m} 3 / \mathrm{s}$ en août (Fig. 2). Ces contrastes temporels sont également inscrits dans l'organisation des écoulements à l'intérieur du bassin.

Fig. 2 : Bilan de l'eau en 1983 dans le bassin du Mouzon

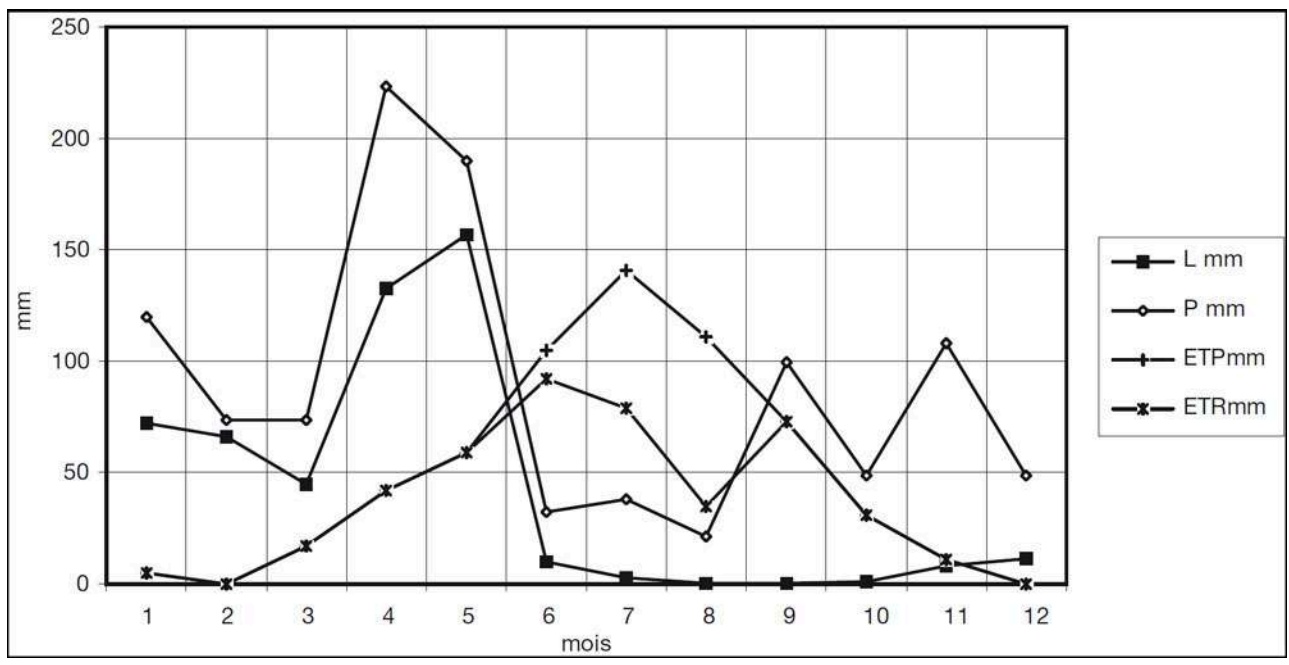

\section{B. Les mesures de terrain}

Trois campagnes de mesures ont été réalisées, au cours des périodes estivales de 1991 et de 1992, sur toutes les artères du réseau hydrographique. Elles comprennent 70 à 75 points de jaugeage ou d'observation, répartis sur tous les cours d'eau (1 point pour 6 $\mathrm{km} 2$ ). Pour chaque campagne, le contexte hydrologique est défini en comparant les débits instantanés mesurés à ceux enregistrés à la station de Villars et aux valeurs de 
débits fréquentiels d'étiage publiées par l'Agence de l'Eau Rhin-Meuse (Catalogue des débits d'étiage).

Les données du tableau 1 montrent que ce contexte correspond à des conditions d'étiage sévères.

Tableau 1 : Débits d'étiage en $\mathrm{m} 3 / \mathrm{s}$ et situation hydrologique des campagnes de mesures

\begin{tabular}{|c|c|c|c|}
\hline Date & $\begin{array}{c}\text { Station de Villars } \\
\text { Qmoy du mois } \\
\text { des mesures }\end{array}$ & $\begin{array}{c}\text { Station de Villars } \\
\text { Qmj du jour } \\
\text { des mesures }\end{array}$ & $\begin{array}{c}\text { Q instantanés } \\
\text { mesurés }\end{array}$ \\
\hline $18 / 6 / 92$ & 0,350 & 0,154 & $\begin{array}{c}\text { Villars: } 0,173, \\
\text { Anger }: 0,058 \\
\text { Ru Etang: } 0,045\end{array}$ \\
\hline $9 / 7 / 91$ & 0,147 & 0,075 & $\begin{array}{c}\text { Villars }: 0,109 \\
\text { Anger }: 0,051 \\
\text { Ru Etang: } 0,025\end{array}$ \\
\hline $20 / 8 / 91$ & 0,129 & 0,073 & $\begin{array}{c}\text { Villars }: 0,071 \\
\text { Anger: } 0,031 \\
\text { Ru Etang }: 0,017\end{array}$ \\
\hline
\end{tabular}

\begin{tabular}{|c|c|c|c|}
\hline $\begin{array}{l}\text { Débits } \\
\text { mensuels } \\
\text { d'étiage }\end{array}$ & & & \\
\hline Station & $Q F 1 / 2$ & $\mathrm{QF} 1 / 5$ & $Q F 1 / 10$ \\
\hline Villars * & $\begin{array}{l}0,194 \\
0,158 * *\end{array}$ & $\begin{array}{l}0,115 \\
h \\
0,084 *\end{array}$ & $\begin{array}{c}0,087 \\
h \\
0,060 \text { * }\end{array}$ \\
\hline Anger" & 0,110 & 0,065 & 0,050 \\
\hline Ru Etang * & 0,045 & 0,025 & 0,020 \\
\hline
\end{tabular}

Extrait des catalogues de débits d'étiage de l'Agence de l'Eau Rhin-Meuse 1954-1974 * et 1971-1990

En 1991, les écoulements indigents sont dus aux précipitations faibles à la fin de l'hiver et au printemps (précipitations mensuelles inférieures à $42 \mathrm{~mm}$ de février à mai). Les débits très bas, mesurés dans le bassin le 9 juillet et le 20 août, pendant la phase de tarissement (Fig. 3), correspondent à des conditions d'étiage sévère, proches des débits mensuels d'étiage, de fréquence $1 / 5$ (juillet 1991) et 1/10 (août 1991).

Fig. 3 : Débits moyens journaliers à la station de Villars au cours des périodes estivales de 1991 et 1992

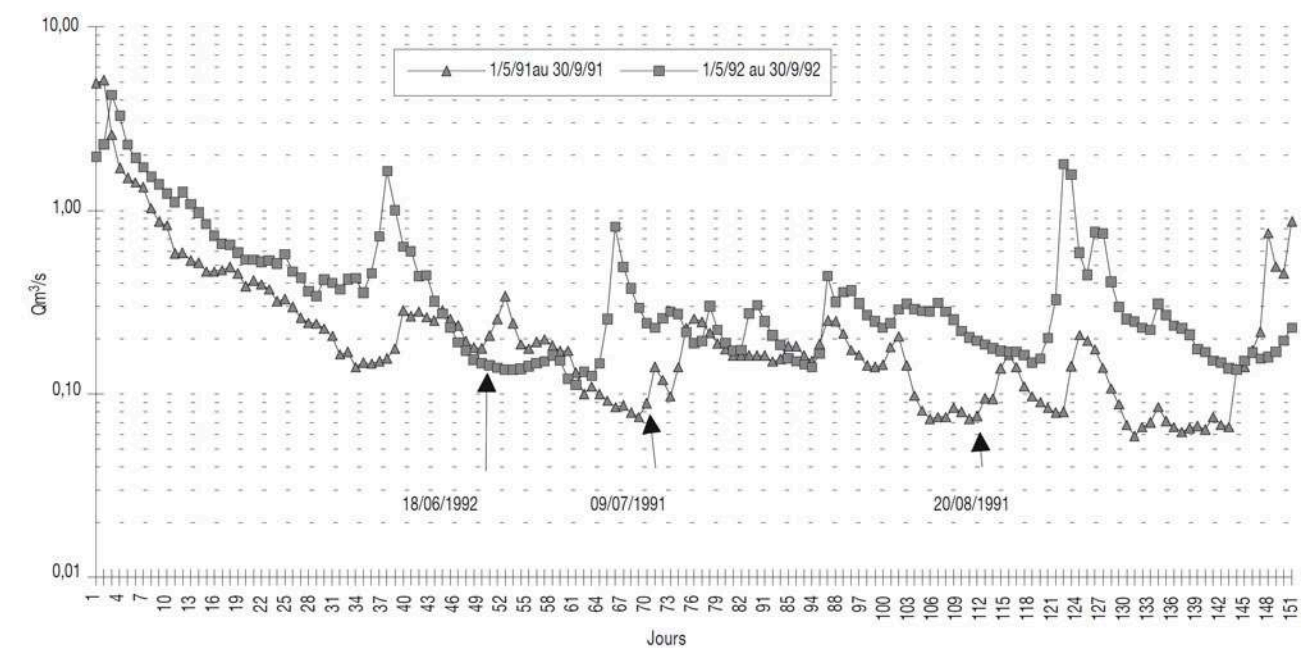

En 1992, les précipitations relativement faibles d'avril à juin (entre 40 et $50 \mathrm{~mm}$ par mois), associées à un hiver modérément pluvieux, ont engendré un étiage précoce. Si le débit moyen mensuel de juin 1992 est encore élevé (0,35 m3/ à la station de Villars), la campagne du 18 juin 1992, menée à la fin d'une période de ressuyage, a permis de mesurer des écoulements dont les volumes sont proches de ceux qui caractérisent les conditions d'étiage bisannuel (Fig. 3).

Les mesures de dureté de l'eau complètent celles des débits pour les campagnes du 18 juin 1992 (ensemble des points de mesures) et du 9 juillet 1991 (mesures réalisées sur le 
Mouzon uniquement) en vue de faciliter l'identification des réservoirs qui participent alors au soutien des écoulements.

\section{Les résultats}

17 Critiqués et analysés dans le détail, ils permettent de définir l'organisation spatiale des écoulements dans le bassin du Mouzon et les différents facteurs qui la déterminent. Les critères de cette définition sont issus de l'interprétation des profils hydrologiques, des cartes de spatialisation des rendements hydrologiques et de la dureté de l'eau.

\section{A. Les profils hydrologiques (Fig. 4)}

18 Ils sont construits pour les cours d'eau principaux du bassin (Mouzon Anger, Bani, Ruisseau de l'Étang). En coordonnées logarithmiques, les points s'alignent sur des segments de droite de pente variable, selon l'organisation spatiale des apports (CORBONNOIS et al. 1999). D'une campagne de mesure à l'autre, ces segments ne restent pas tous parallèles et sont fonction des capacités de soutien des aquifères. Les profils du Mouzon et de l'Anger qui drainent les mêmes ensembles de roches, se ressemblent avec des secteurs amont à sec et un débit relativement abondant à partir des points M5 et A5 qui contrôlent des bassins de l'ordre de $20 \mathrm{~km} 2$. L'eau provient de la nappe des calcaires du Muschelkalk recoupée par les ruisseaux en fond de vallée, libre (Mouzon) ou semicaptive, sous un mince recouvrement d'argiles du Keuper (Anger). Vers l'aval, dans la traversée des grès du Rhétien et des calcaires à gryphées (Lias inférieur) le débit des cours d'eau augmente d'un tiers (entre M 14 et M25, et A5 et A8). Mais cet apport se réduit à $1 / 5$ sur le Mouzon en août 1991. Ces niveaux, qui livrent alors moins d'eau, déterminent sur le profil hydrologique une modification de la pente du segment de droite correspondant à ce secteur. Dans la traversée des calcaires du Bajocien, les débits diminuent. On mesure une infiltration de l'eau dans le substratum, qui grandit (déplacement vers l'amont du point de rupture du profil) avec l'abaissement du niveau piézométrique au cours de l'étiage. 
Fig. 4 : Profils hydrologiques des cours d'eau du bassin du Mouzon
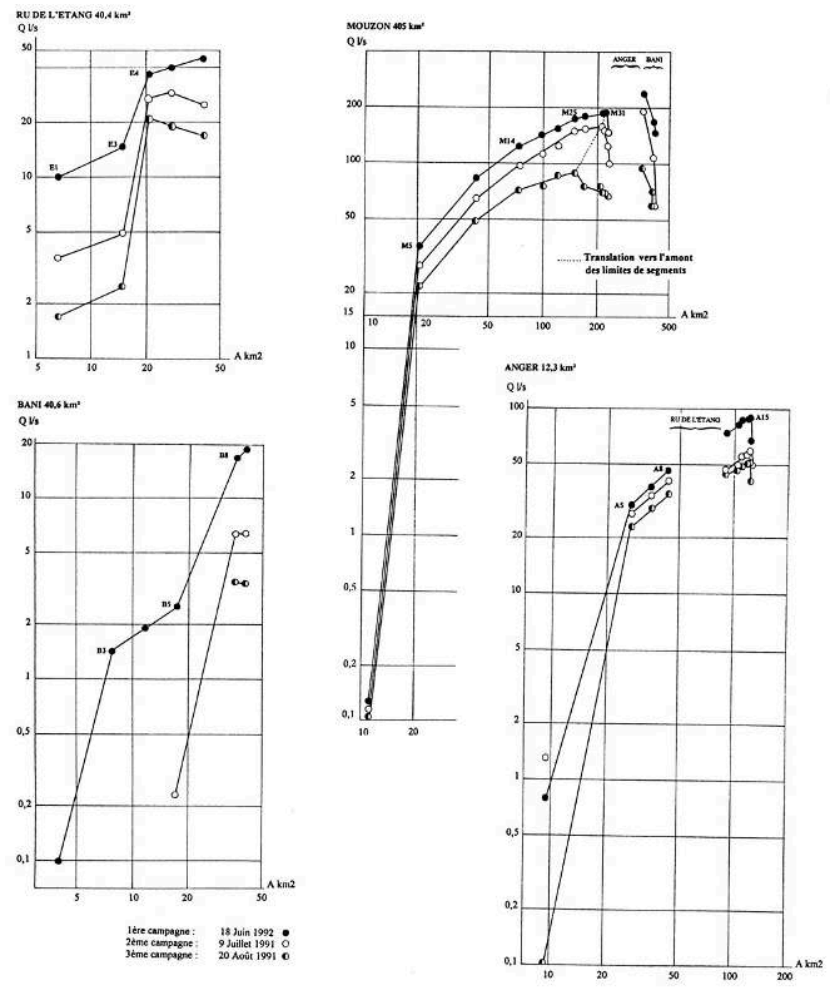

Le Bani et le ruisseau de l'Étang, qui ne drainent pas les sédiments du Trias, ont des profils différents. En étiage sévère, le Bani s'assèche sur les trois quarts de son cours amont qui draine un substratum sans réserve souterraine. Le ruisseau de l'Étang écoule à l'amont l'eau de la nappe des grès rhétiens qui soutient bien le débit. Vers l'aval, celui-ci a tendance à diminuer lorsque le substratum devient marneux et ne livre pas d'eau. La réduction des volumes écoulés peut alors être attribuée aux prélèvements évaporatoires, perceptibles lorsque les débits sont faibles.

Ces profils mettent finalement en évidence la variabilité dans l'espace et dans le temps de la capacité de soutien des nappes aux écoulements de surface. C'est une des caractéristiques du bassin du Mouzon qui apparaît également sur les cartes des rendements hydrologiques.

\section{B. Spatialisation des rendements hydrologiques (Fig. 5)}

Le rendement hydrologique correspond à un débit spécifique calculé entre deux points de mesure (rapport entre l'écart de débit et l'écart de surface correspondante). Dans le bassin du Mouzon, les rendements ne s'élèvent jamais au-dessus de $5 \mathrm{l} / \mathrm{s} / \mathrm{km} 2$. Trois classes principales, déterminées selon les différents ensembles lithologiques, ont été représentées sur les cartes. Ces dernières montrent qu'entre juin 1992, juillet et août 1991, les secteurs producteurs d'eau se rétrécissent, en accord avec le fonctionnement hydrodynamique des nappes souterraines. Du fait de leur faible capacité et de l'absence de recharge, les réserves diminuent ou tarissent lorsque la sévérité de l'étiage augmente, comme l'ont déjà montré les profils. 
Fig. 5 : Spatialisation des rendements hydrologiques

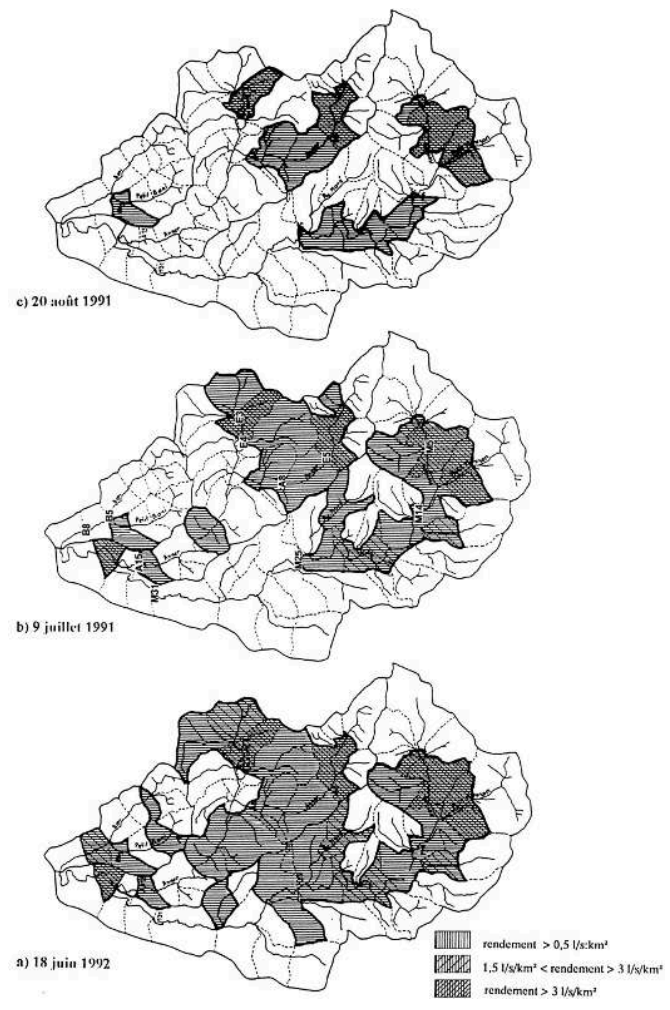

En juin 1992, la plupart des réservoirs contiennent encore un peu d'eau et les troisquarts du bassin ont un rendement égal ou supérieur à $0,5 \mathrm{l} / \mathrm{s} / \mathrm{km} 2$. Les secteurs à sec ou faiblement alimentés, se situent en bordure Sud-Ouest (affluents de rive gauche du Mouzon) et Nord-Est du bassin, où affleurent des matériaux à dominante imperméable du Lias supérieur, surmontés vers l'aval par les calcaires du Bajocien. Au Sud-Est du bassin, où affleurent les marnes du Keuper, les rendements très faibles sur les branches amont du réseau hydrographique, augmentent vers l'aval avec l'approfondissement des vallées, mais sans qu'il n'y ait de changement lithologique (Mouzon entre Martigny-lesBains et Rocourt, Anger entre Crainvilliers et la Vacheresse).

En juillet 1991, ces secteurs (rendement supérieur à $0,5 \mathrm{l} / \mathrm{s} / \mathrm{km} 2$ ) ne concernent plus qu'un tiers du bassin. Les conditions d'étiage plus sévères se manifestent par une diminution des rendements dans l'aval du bassin, à l'exception de deux secteurs isolés correspondant à l'affleurement des grès médioliasiques (Anger aval) et des calcaires du Bajocien.

En août 1991, les secteurs encore fonctionnels qui correspondent aux réservoirs encore riches en eau et contenant des réserves pérennes, se maintiennent dans la partie SudEst du bassin. L'eau est contenue dans les couches du Muschelkalk supérieur (calcaires à cératites et dolomie de Vittel) surmontées par les marnes du Keuper minces et disloquées, ainsi que dans les grès du Rhétien, en général faiblement aquifères en Lorraine (CEGUM 1980), avec des rendements supérieurs à $3 \mathrm{l} / \mathrm{s} / \mathrm{km} 2$.

A l'aval du bassin, l'aquifère du Bajocien n'est plus que ponctuellement productif (bassin du Bani). Ailleurs, les réserves sont épuisées.

Ainsi, en étiage sévère, de grandes parties du bassin sont à sec. Les nappes qui participent encore aux écoulements sont semi-captives à l'amont du bassin et recoupées en fond de vallée par le réseau hydrographique conséquent. Les secteurs 
concernés ont des rendements compris entre 3 et $5 \mathrm{l} / \mathrm{s} / \mathrm{km} 2$ selon les campagnes. Le stock d'eau échappe à l'égouttage par gravité qui accélère la vidange des nappes perchées et explique, en partie, l'épuisement de celles habituellement contenues dans les calcaires du Bajocien (Corbonnois, 1996). Dans le bassin du Mouzon, ce sont de médiocres réservoirs (capacité de stockage réduite du fait de fissuration et fracturation d'origine tectonique) par lesquels l'eau transite rapidement. La nappe n'alimente que des sources temporaires localisées sur les versants, au contact des marnes du Toarcien, à sec au moment des campagnes de mesures. À l'extrême aval du bassin, où les vallées sont entièrement inscrites dans le calcaire, l'eau circule en profondeur dans le sens du pendage et rejoint souterrainement la Meuse, en amont ou en aval de Neufchâteau (Maubeuge, 1980). Par ailleurs, la présence d'une forêt de feuillus qui occupe en quasitotalité les plateaux calcaires, contribue à diminuer la quantité d'eau disponible à l'infiltration et à augmenter les prélèvements évapo-transpiratoires (Humbert, Najjar, 1992).

\section{Origine et dynamique des apports souterrains}

À chaque point, la mesure de la dureté de l'eau (dosage du calcium et du magnésium par compléxométrie) permet de préciser son origine. Les résultats obtenus pour la campagne du 18 juin 92, dans l'ensemble du bassin, et pour celle du 9 juillet 91 , sur le Mouzon uniquement, font apparaître des contrastes spatiaux marqués en liaison avec la diversité lithologique (Ramon et Gully, 1979). Les mesures se rapportant aux branches amont du réseau hydrographique (points contrôlant de petites surfaces de bassin versant, inférieures à $40 \mathrm{~km} 2$ ), permettent d'identifier l'eau selon les ensembles lithologiques principaux (tableau 2). Vers l'aval, les eaux se mélangent (dilution, précipitation, ou nouveaux apports d'eau différemment chargés).

Tableau 2 : Dureté de l'eau et lithologie correspondante

\begin{tabular}{|c|c|}
\hline Dureté $\left(\mathrm{TH}^{*} 10\right.$ en $\left.\mathrm{mg} / \mathrm{l}\right)$ & Ensemble lithologique \\
\hline Inférieure à. 200 & Grès du Rhétien \\
\hline De 200 à 320 & Calcaires à gryphées, calcaires du Bajocien et grès médioliasiques \\
\hline De 320 à 500 & Marne du Lias supérieur \\
\hline De 500 à 800 & $\begin{array}{l}\text { Calcaires et dolomies du Muschelkalk supérieur et eaux mélangées des } \\
\text { secteurs de transit aval }\end{array}$ \\
\hline De 800 à 1000 & Eau mélangée des secteurs de transit amont \\
\hline Supérieure à 1000 & $\begin{array}{l}\text { Marnes du Keuper et Lettenhkohle, riches en anhydrite, gypse } \\
\text { et dolomie }\end{array}$ \\
\hline
\end{tabular}

Il est possible de définir des types d'écoulement à partir du rendement hydrologique et la dureté de l'eau. Une corrélation logarithmique permet de regrouper en 8 nuages, les différents points de mesures (Fig. 6). 
Fig. 6 : Rendements hydrologiques et teneurs en $\mathrm{CaCO} 3 \mathrm{mg} / \mathrm{l}$ équivalent $\left(\mathrm{TH}^{\circ} \mathrm{F}^{\star} 10\right)$

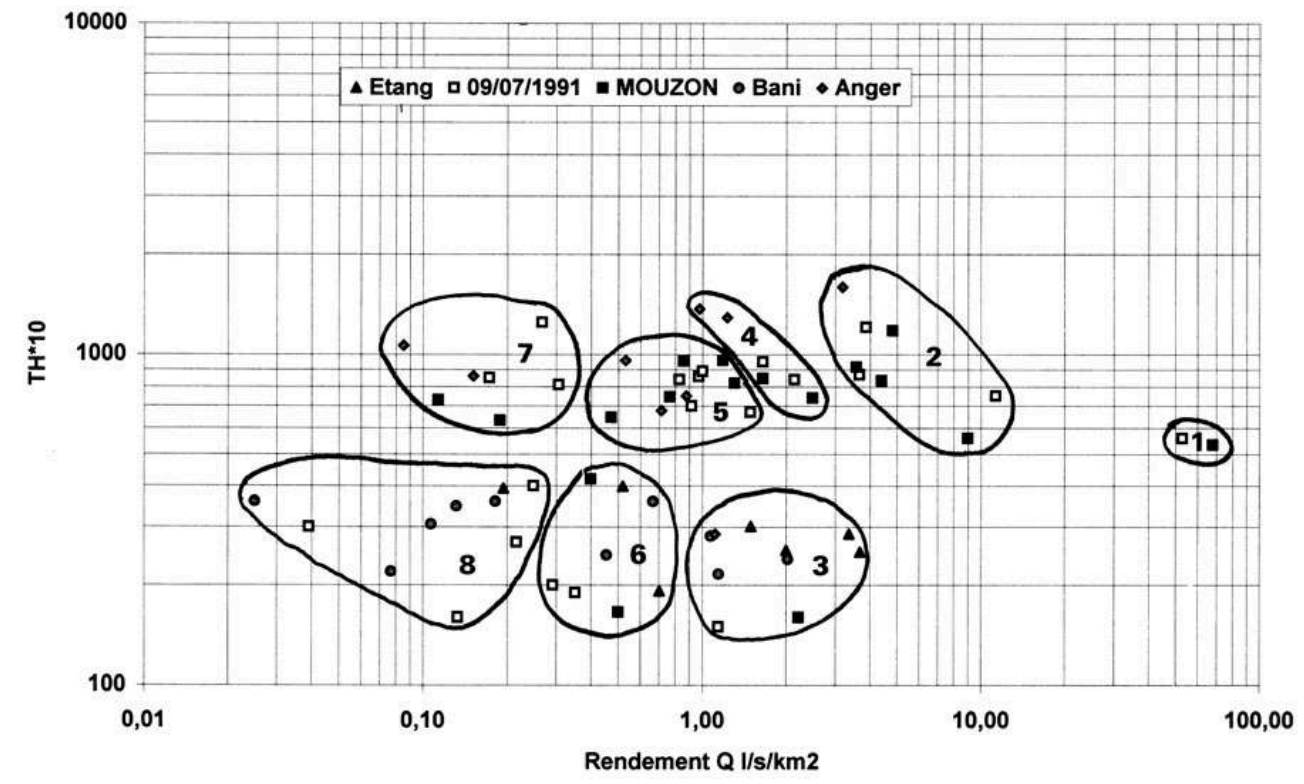

Chaque nuage correspond à un mode de production d'eau défini par la nature du réservoir et la dynamique des apports, selon les ensembles lithologiques, mais également pour un même ensemble, selon la tectonique et les conditions géomorphologiques. Les différents types sont cartographiés sur la figure 7 (résultats des campagnes du 18 juin 1992 et du 9 juillet 1991).

Les secteurs les plus productifs correspondent au drainage des nappes des calcaires du Muschelkalk et des grès du Rhétien mais regroupent plusieurs types d'écoulements :

Les types 1 et 2 regroupent les secteurs où l'eau est livrée par la nappe des calcaires et dolomies du Muschelkalk supérieur, modérément minéralisée $(550 \mathrm{ml} / \mathrm{l}$ équivalent CaCO3), et recoupée par le Mouzon seul à Martigny-les-Bains. Son rendement élevé est probablement influencé par des apports karstiques (type 1). Vers l'aval, cette nappe est semi-captive sous les marnes du Keuper, pauvres en eau. Elle alimente alors le réseau hydrographique en fond de vallée, par drainance ascendante, là où ces marnes sont minces. Au cours de son transit, l'eau de nappe dissout les niveaux de sel et surtout de gypse contenus dans le Keuper qui livrent du sulfate de calcium. On définit ainsi un deuxième type d'écoulement qui comprend le Mouzon amont, le Petit Mouzon ainsi que l'Anger amont (secteur A5). Les rendements soutenus (3 à $5 \mathrm{l} / \mathrm{s} / \mathrm{km} 2)$ sont associés à de fortes teneurs en éléments dissous (plus de $1000 \mathrm{mg} / \mathrm{l}$ équivalent CaCO3).

Le type 3 correspond à certains secteurs du Bani où affleurent les grès médioliasiques et les calcaires du Bajocien. Mais il regroupe également des ruisseaux alimentés par la nappe des grès du Rhétien aux eaux faiblement minéralisées (moins de $320 \mathrm{mg} / \mathrm{l}$ ). Dans les bassins du ruisseau de l'Étang et du ruisseau Moré, cette nappe a un bon rendement (1 à 4 l/s/km2) du fait des conditions locales propices : affleurement des grès en fond de vallée, apports supplémentaires d'eau stockée dans les calcaires à gryphées sus-jacents, dont la nappe communique par drainance, à travers les minces argiles de Levallois, avec celle des grès. Ces communications sont facilitées dans les secteurs fracturés. Les grès du Rhétien participent également au soutien des débits du Mouzon et de l'Anger dans la partie médiane du bassin. Mais le réservoir alors perché, livre moins d'eau (type 4 entre 1 et $2,5 \mathrm{l} / \mathrm{s} / \mathrm{km} 2$, type 5 entre 0,5 et $1,6 \mathrm{l} / \mathrm{s} / \mathrm{km} 2$ ). Celle-ci dilue cependant les fortes duretés acquises dans la partie amont du bassin. 
Les autres types d'écoulement ont de faibles rendements hydrologiques :

- le type 6 correspond à de petits ruisseaux, peu incisés et mal alimentés $(0,3$ à $0,7 \mathrm{l} / \mathrm{s} / \mathrm{km} 2)$, drainant les mêmes ensembles lithologiques que le type 3 (duretés proches) ;

- le type 7 regroupe les écoulements en cours de tarissement qui parcourent les marnes ;

- le type 8 correspond aux parties aval des bassins du Mouzon et de l'Anger où les écoulements de surface tendent à s'infiltrer dans les calcaires du Bajocien.

Fig. 7 : Cartographie des rendements hydrologiques et des duretés associées

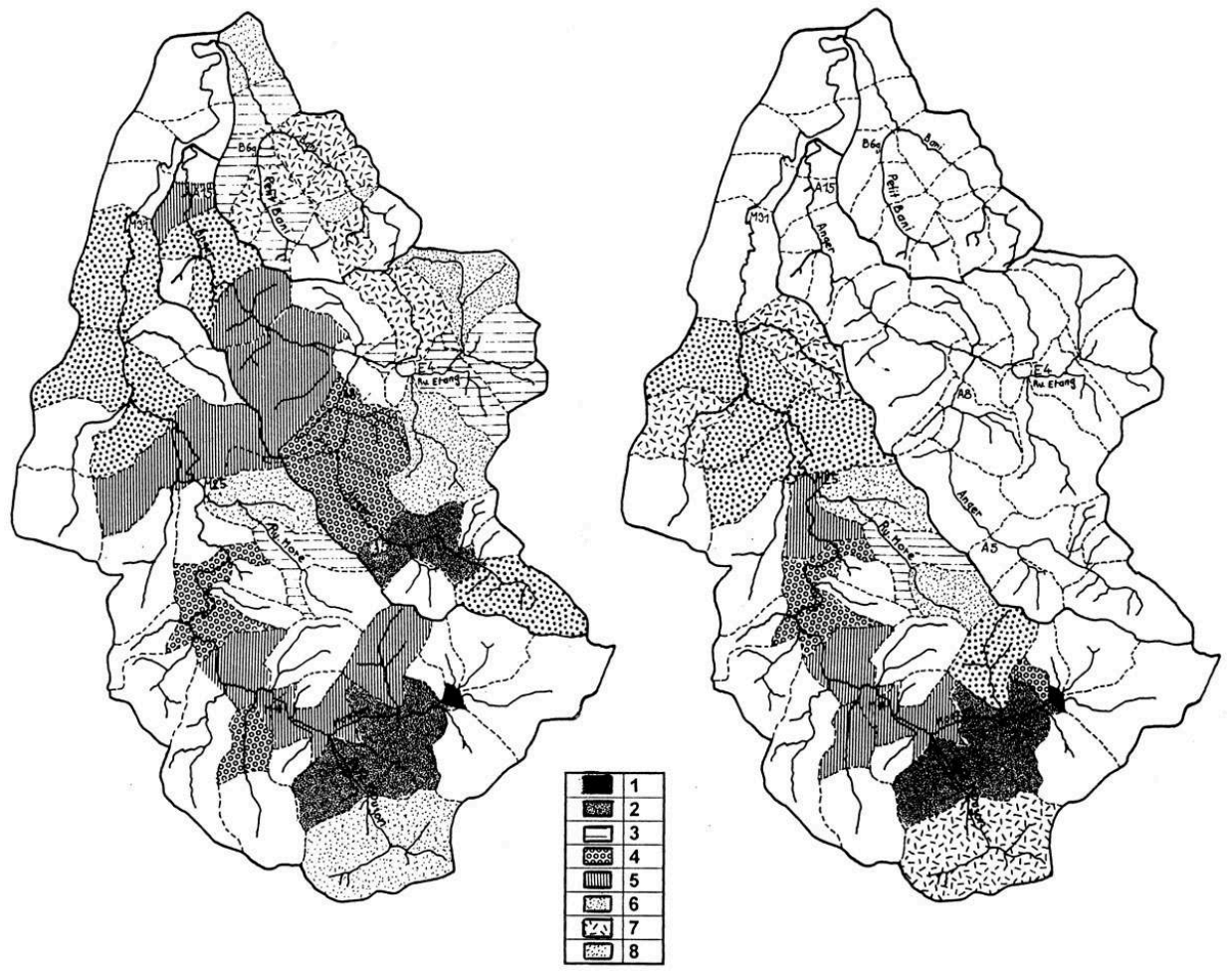

Légende : Numéro des nuages de points de la figure 6.

\section{Conclusion}

Le bassin du Mouzon se prête finalement à une bonne détermination des facteurs qui influencent les écoulements en étiage. Il présente une série de niveaux aquifères minces et plutôt pauvres en eau. Les résultats obtenus ne sont toutefois pas vraiment originaux (Lambert, 1995). Il s'agit pourtant d'un des rares bassins, où en plus des facteurs structuraux et géomorphologiques, habituellement évoqués pour expliquer les écoulements en étiage, il soit possible de percevoir l'impact des prélèvements évaporatoires qui réduisent les débits et le rôle du couvert végétal qui participe à l'épuisement précoce des réserves souterraines.

L'organisation des écoulements mise en évidence répond ainsi à une association de facteurs se rapportant à diverses échelles. Les facteurs régionaux expliquent les grands traits de la structure, la disposition du réseau hydrographique (conséquent dans le bassin du Mouzon) et les caractéristiques du climat. Les précipitations influencent l'état des réserves au début de la période d'étiage, les prélèvements évaporatoires accélèrent, au cours des étés secs, le tarissement des écoulements. Les facteurs locaux, 
fracturations, déformations souples, couvert végétal, introduisent des variantes dans les rendements hydrologiques. Celles-ci expliquent les différences d'apports livrés par les calcaires du Bajocien ; absorbants dans le Mouzon et l'Anger aval (effet de soutirage vers le bassin de la haute Meuse), ils livrent encore de l'eau dans le bassin du Bani, ou celui de l'Aroffe situé à $30 \mathrm{~km}$ au Nord-Est (Corbonnois, 1996). Ces variantes expliquent également les rendements plus élevés des grès du Rhétien dans le bassin versant du ruisseau de l'Étang. Les facteurs stationnels : profondeur des entailles, caractère perché ou non des niveaux aquifères, mais également, existence d'un colmatage discontinu d'alluvions, expliquent les différences de volume des stocks d'eau dont il faut tenir compte dans le fonctionnement hydrodynamique des bassins. Ce sont ces facteurs associés qui gèrent l'écoulement en étiage. Mais tous les bassins ne se prêtent pas à leur détermination. Certains facteurs sont masqués lorsque l'eau reste suffisamment abondante au cours de l'été (précipitations estivales) et que les apports du substratum compensent partiellement les prélèvements évapo-transpiratoires et atténuent l'impact toujours ténu du couvert végétal dans le Nord-Est de la France.

Finalement, nos résultats, qui mènent à une lecture assez fine des conditions de vidange des réserves stockées sous la surface, restent malgré tout, tributaires de la densité des mesures de terrain. La démarche mise en œuvre permet cependant d'appréhender dans le détail le comportement hydrogéologique du bassin en étiage, que la seule interprétation des données à l'exutoire ne pouvait permettre.

\section{BIBLIOGRAPHIE}

Arts I., Francois D. (1998). - Profils hydrologiques du bassin de la Meurthe. Proposition d'une typologie. Mosella, Tome XVIII, Nº 1-2, 169-192.

Castany G. (1982). - Principes et méthodes de l'hydrogéologie, Paris, Dunod.

CEGUM/Agence de l'eau Rhin-Meuse (1992). - Catalogue des débits d'étiage de la Meuse. Campagne 1991 et 1992, Bassin du Mouzon. Rapport d'étude.

CEGUM/SRAEL (1980). - Synthèse hydrogéologique des départements de la Moselle et de la Meuthe-etMoselle. Rapport d'étude.

Corbonnois J. (1996). - Organisation spatiale des débits et des éléments dissous dans les cours d'eau en période estivale. Bassins versants de la Chée et de l'Aroffe amont (Lorraine France).

Hommes et Terres du Nord, 1/96, pp. 21-28.

Corbonnois J., Francois D., Arts I., Sary M., Devos A., Decloux J.P., Gille E., Gamez P. (1999). Répartition des écoulements en basses eaux. Aspects méthodologiques et applications. À paraître dans Rapport quadriennal CNFSH.

Cosandey C. (1983). - Recherches sur les bilans de l'eau dans l'Ouest du massif Armoricain. Thèse de doctorat d'état. Paris Sorbonne, $515 \mathrm{p}$.

Dacharry M. (1975). - Sur les fléchissements des débits estivaux de deux rivières du Massif Central français, la Senouire et l'llanche, affluent et sous-affluent de l'llier. RGE, 1-2, pp 89-99. 
Francois D., Gille E., Zumstein J.F. (1993). - Analyse des séries chronologiques et applications aux données hydroclimatologiques. In Hommage à René Frecaut, L'Eau, la Terre, les Hommes, PUN, Nancy.

Francois D., Sary M., Auer J.Cl., Zumstein F. (1994). - Étude méthodologique des débits d'étiage. Rapport AERM/ CEGUM.

Humbert J., Najjar G. (1992). - Influence de la forêt sur le cycle de l'eau en domaine tempéré. Une analyse de la littérature francophone. CEREG, Université Louis Pasteur, Strasbourg.

Lambert R. (1994). - De la crue du débit de base des rivières du bassin garonnais d'août à octobre alors que perdure la sècheresse atmosphérique. Dossier de la $R G A, \mathrm{~N}^{\circ} 12, \mathrm{pp} .117-123$.

Lambert R. (1995). - Géographie du cycle de l'eau. Presses universitaires du Mirail. Coll. Amphi 7, Géographie. 440 p.

Lelong F., Andreux M., Amiot M., Lepiller M. (1994). - Influence du sous-sol, du sol et de son utilisation sur la chimie des eaux souterraines et de surface en climat tempéré. Journées d'études de l'AIDEC, Dijon 17-16 mars 1994. Cahier N 32, pp. 59-65.

Maire G. (1977). - Étude préliminaire sur les étiages dans le bassin de la Bruche : recherche d'une zonation des débits spécifiques de basses eaux d'octobre 1976 en fonction de diverses caractéristiques physico-géographiques des bassins versants. Recherches géographiques à Strasbourg. $\mathrm{N}^{\circ} 4$.

Martin Ph. (1991). - Quantification des flux carbonatés exportés par les aquifères de la Sainte Baume (Var, France) et estimation de la dissolution spécifique actuelle sur ce massif. Etudes de géographie physique. Travaux Nº XX. Univ. d'Aix-Marseille II, URA 903, CNRS, pp. 25-36.

Maubeuge P.L. (1980). - Le problème des pertes de la Meuse et de l'hydrologie des environs de Neufchâteau (Vosges). Académie et Société Lorraine des Sciences. Tome 19, № 4.

Mazrag Z. (1990). - Hydroclimatologie du bassin versant du Mouzon. DEA, CEGUM, Université de Metz.

Merot P. (1988). - Les zones de sources à surface variable et la question de leur localisation. Hydrologie Continentale, 3 (2), pp. 105-115.

Rambert B. (1971). - Application de l'analyse des hydrogrammes des cours d'eau à l'évaluation de l'écoulement souterrain. Rapport BRGM-SGN 389 Hyd., 34 p.

Ramon S., Gully J. (1979). - Qualité des eaux souterraines dans les départements de la Meuse et de la Meurthe et Moselle. Mosella, Tome IX, N 3-4.

Sary M. (1993). - Signification du profil hydrologique de basses eaux : cas de la Plaine dans le Massif Vosgien. In Hommage à René Frecaut, L'Eau, la Terre, les Hommes. PUN, Nancy.

Zumstein J.F. (1976). - Contribution à l'élaboration des cartes hydrologiques, cartes des étiages mensuels des bassins de la Meuse et de la Moselle de 1969 à 1973. La Houille Blanche $\mathrm{N}^{\circ} 6$.

Notices et cartes géologiques de Vittel, Bourmont, Chatenois, Neufchâteau.

\section{RÉSUMÉS}

En étiage, les apports d'eau livrés par les nappes souterraines soutiennent les écoulements de surface. Dans le bassin du Mouzon (415 km2), ces apports sont identifiés par des mesures de débits complétées par celles des éléments dissous contenus dans les eaux de surface. Les résultats obtenus sont spatialisés. On montre ainsi que les volumes d'eau contenus dans les réservoirs les 
plus productifs sont déterminés par la lithologie, mais également par la tectonique qui guide l'écoulement des nappes. Par ailleurs, les conditions de drainage des nappes par le réseau hydrographique (disposition du réseau hydrographique par rapport au pendage des couches, profondeur de l'incision par les cours d'eau, profondeur des niveaux aquifères), est également un élément déterminant dans l'alimentation en eau du réseau hydrographique. Les caractéristiques du bassin du Mouzon permettent de mettre clairement en évidence ces différents éléments qui participent à l'organisation spatiale des écoulements en étiage.

In low discharge periods, the water flowing from the underground waterlevels, feeds surface discharge. In the Mouzon basin (415 square $\mathrm{km}$ ), these supplies are identidied by measures of flows and of dissolved material. Then, results are spacially analysed. We can prove that volumes of water coming from the more productive reservoirs are determined by lithology and tectonics. Moreover, conditions of the drainage by the hydrological network (layout of this network in relation to disp of strata, depth of water incision and underground waterlevels), is also an important factor in water supply to the hydrographical network. The characteristics of the Mouzon basin show clearly the different factors that contribute to the spatial organization of water flows during low water periods.

Während der Niedrigwasserzeit ist der Abfluss vom Grundwasser ernährt. Im Einzugsgebiet des Mouzon ist dieser Beitrag durch Abfluss und Lôsungsinhalt Gemässe identifiziert. Die Resultaten sind kartographiert. Man zeigt so, dass die unterirdischen Wassermenge von der geologische Stuktur abhängt, die dass Wasser unter der Fläche leitet. Die geomorphologischen Factoren spielen auch eine Rolle in der Abflussbildung. Das Einzugsgebiet des Mouzon ist ein gutes Beispiel für diese verchiedenen Elementen hervorzuheben, die an der Wasserverteilung teilnehmen.

\section{INDEX}

Schlüsselwörter : Grundwasser, hydrologische Karte, Lothringen, Niedrigwasserzeit

Mots-clés : débit, étiage, Lorraine, nappe, spatialisation

Keywords : flow spatialisation, Lorraine, low water, underground waterlevel

\section{AUTEUR}

JEANINE CORBONNOIS

Centre d'Études Géographiques de l'Université de Metz - UFR Sciences Humaines et Arts - Ile du Saulcy 57045 Metz Cedex 01 